Mon. Not. R. Astron. Soc. 000,113(2006) Printed 20 August $2019 \quad$ (MN LATEX style file v2.2)

\title{
X-ray emissions from two-temperature accretion flows within a dipole magnetic funnel
}

\author{
Curtis J. Saxton ${ }^{1}$, Kinwah Wu ${ }^{1,2}$, João. B. G. Canalle ${ }^{3}$, Mark Cropper ${ }^{1}$ \\ \& Gavin Ramsay ${ }^{1,4}$ \\ 1 Mullard Space Science Laboratory, University College London, Holmbury St Mary, Dorking, Surrey RH5 6NT, England \\ 2 TIARA, Department of Physics, National Tsing Hua University, Hsinchu 300, Taiwan \\ 3 State University of Rio de Janeiro, Rua São Francisco Xavier, 524/3023-D, CEP 20559-900, Rio de Janeiro, RJ, Brazil \\ 4 Armagh Observatory, College Hill, Armagh BT61 9DG, N. Ireland
}

Accepted --. Received --; in original form --

\begin{abstract}
We investigate the hydrodynamics of accretion channelled by a dipolar magnetic field (funnel flows). We consider situations in which the electrons and ions in the flow cannot maintain thermal equilibrium (two-temperature effects) due to strong radiative loss, and determine the effects on the keV X-ray properties of the systems. We apply this model to investigate the accretion shocks of white dwarfs in magnetic cataclysmic variables. We have found that the incorporation of two-temperature effects could harden the keV X-rays. Also, the dipolar model yields harder X-ray spectra than the standard planar model if white dwarf is sufficiently massive $\left(\gtrsim 1 M_{\odot}\right)$. When fitting observed $\mathrm{keV}$ X-ray spectra of magnetic cataclysmic variables, the inclusion of two-temperature hydrodynamics and a dipolar accretion geometry lowers estimates for white-dwarf masses when compared with masses inferred from models excluding these effects. We find mass reductions $\lesssim 9 \%$ in the most massive cases.
\end{abstract}

Key words: accretion, accretion discs - hydrodynamics — shock waves - stars: binaries: close - stars: white dwarfs - X-rays: binaries

\section{INTRODUCTION}

Field-channelled accretion occurs in a variety of stellar systems, from young stellar objects (e.g. Königl 1991; Hartman et al. 1994; Gullbring et al. 2000; Lamzin et al. 2001; Romanova et al. 2003; Stelzer \& Schmitt 2004; Gregory et al. 2006) to compact stars (e.g. Elsner \& Lamb 1977; Ghosh \& Lamb 1978; Arons 1993; Lovelace et al. 1995; Li et al. 1996; Li \& Wilson 1999; Kryukov et al.|2000; Koldoba et al. 2002; Toropina et al. 2003; Canalle et al. 2005), where the magnetic stress of the accretor exceeds the ram pressure of the accretion flow. For accretion onto white dwarfs in magnetic cataclysmic variables (mCVs) Warner 1995; Cropper 1990), the magnetic field not only directs the flow but could also dictate the radiative-loss processes (see e.g. Wu et al. 2003). This, in turn, alters the flow hydrodynamics, leading to observable consequences, for example, in the optical/IR polarisation and keV X-ray spectra of the system.

The keV X-ray emitting regions of $\mathrm{mCV}$ s are usually located at the magnetic field footpoints on the white-dwarf surface. Here an optically thin plasma slab heated by a strong shock, at which the supersonic flows in the upstream region decelerate abruptly to attain subsonic speeds. Typically, the shock temperature is $T \approx 3 G M_{\mathrm{w}} m_{\mathrm{H}} / 8 k R_{\mathrm{w}} \sim$ $10-50 \mathrm{keV}$ (where $G$ is the gravitational constant, $k$ is the Boltzmann constant, $m_{\mathrm{H}}$ is the hydrogen atomic mass, $M_{\mathrm{w}}$ is the white-dwarf mass, $R_{\mathrm{w}}$ is the white-dwarf radius). The shock-heated flow is cooled by emission of free-free and line X-rays, and optical/IR cyclotron radiation.

The post-shock regions of white dwarfs in $\mathrm{mCVs}$ are stratified in temperature and density, and the flow structures are in general well described by Aizu-type models (Aizu 1973; Chevalier \& Imamura 1982; Wu 1994; Wu et al. 1994; Cropper et al. 1999; Wu 2000). These models assume that the electron and ions are in thermal equilibrium, via collisions, and share the same local temperature. (Hereafter, these models are denoted as standard one-temperature (1T) models.) The standard $1 \mathrm{~T}$ models have successfully explained the line features in the $\mathrm{keV} \mathrm{X}$-ray spectra of $\mathrm{mCVs}$. However using the standard $1 \mathrm{~T}$ models to generate spectral fits for mCVs tends to require white-dwarf masses systematically higher than the masses measured by other techniques (see Ramsay et al. 1998; Ramsay 2000). This has led to questions on the validity the $1 \mathrm{~T}$ approximation.

It has long been recognised (Fabian et al. 1976; 
King \& Lasota 1979; Lamb \& Masters 1979) that the electrons and ions in the post-shock flows of mCVs might be unable to maintain thermal equilibrium solely via electronion collisions (see also Imamura 1981; Imamura et al. 1987, 1996; Woelk \& Beuermann 1996; Saxton \& Wu 1999, 2001; Fischer \& Beuermann 2001). The departure of the electron temperature from the ion temperature can be severe if the radiative-loss timescale of the flow is much shorter than the dynamical timescale. In such situations, two-temperature $(2 \mathrm{~T})$ effects on the X-ray spectra are no longer negligible. Two-temperature effects on X-ray spectra of mCVs were previously investigated assuming planar stratified flow (Saxton et al. 2005). However, the accretion flows in mCVs are not planar. There is evidence that the accretion flows form curved funnel/curtain-like columns along the magnetic field lines (Ferrario 1996; Heerlein et al. 1999). The hydrodynamics in funnel flow and in planar are qualitatively different, especially for tall post-shock columns where a variation of the gravitational potential is significant (Canalle et al. 2005).

Here we investigate $2 \mathrm{~T}$ accretion in $\mathrm{mCVs}$ where the flows are guided by the white-dwarf magnetic field geometry. This work is a generalisation of both the $2 \mathrm{~T}$ planar flow calculations of Saxton et al. (2005) and the 1T dipole-field channelled flow calculations of Canalle et al. (2005). We organise the paper as follows. In $\S 2$ we present the model assumptions and the hydrodynamic formulation; in $\S 3$ we show the hydrodynamical structures and X-ray spectra obtained from the calculations and discuss the properties of the flows (and their dependence on system paramters). In $\S 4$ we summarise the major findings.

\section{HYDRODYNAMIC FORMULATION}

For a gas with adiabatic index $\gamma$, density $\rho$, velocity $\mathbf{v}$ and pressure $P$, subject to a gravitational field $\mathbf{g}$, the general hydrodynamic equations for conservation of mass, momentum and energy can be expressed as

$\frac{\partial \rho}{\partial t}+\nabla \cdot \rho \mathbf{v}=0$

$\frac{\partial}{\partial t} \rho \mathbf{v}+\nabla \cdot \rho \mathbf{v} \mathbf{v}+\nabla P=\rho \mathbf{g}$,

and

$$
\left(\frac{\partial}{\partial t}+\mathbf{v} \cdot \nabla\right) P-\frac{\gamma P}{\rho}\left(\frac{\partial}{\partial t}+\mathbf{v} \cdot \nabla\right) \rho=-(\gamma-1) \Lambda,
$$

where $\Lambda$ is the volumetric energy loss rate due to radiative cooling. Emission by electrons far exceeds emission by the ions. Unlike Canalle et al. (2005), who assumed temperature equilibrium between electrons and ions, we will consider cases where electron radiative cooling is significant compared to collisional heating by the ions. This entails a separate energy equation for the electron sub-fluid,

$$
\left(\frac{\partial}{\partial t}+\mathbf{v} \cdot \nabla\right) P_{\mathrm{e}}-\frac{\gamma P_{\mathrm{e}}}{\rho}\left(\frac{\partial}{\partial t}+\mathbf{v} \cdot \nabla\right) \rho=(\gamma-1)(\Gamma-\Lambda),
$$

where $P_{\mathrm{e}}$ is the electron partial pressure. The electron cooling function, $\Lambda=\Lambda\left(\rho, P_{\mathrm{e}}\right)$, and the collisional heating function, $\Gamma=\Gamma\left(\rho, P, P_{\mathrm{e}}\right)$, depend on local hydrodynamic variables (Spitzer 1962; Rvbicki \& Lightman 1979; Imamura et al. 1996).
We consider accretion flows that are channelled by a dipolar magnetic field centred on the accreting stellar object. The coordinate $w$ measures paths along a magnetic field line towards the accretor. A transverse coordinate $u=\sin ^{2} \theta_{*}$ is related to the colatitude of the accretion hot-spot $\left(\theta_{*}\right)$. The azimuthal angle $\varphi$ is the same as in cylindrical coordinates. We seek stationary solutions for the flow structure, so we omit temporal derivatives from (1)-(4). We recast the equations in curvilinear coordinates, $(\hat{\boldsymbol{u}}, \hat{\boldsymbol{w}}, \hat{\boldsymbol{\varphi}})$. For flows along the field line, there is no velocity component in the $\hat{\boldsymbol{u}}$ or $\hat{\boldsymbol{\varphi}}$ directions. Then the hydrodynamic equations for mass flux, momentum flux, total energy and electron energy simplify to

$\frac{\partial}{\partial w} h_{1} h_{3} \rho v=0 \Rightarrow h_{1} h_{3} \rho v=C$,

$\frac{v}{h_{2}} \frac{\partial v}{\partial w}+\frac{1}{h_{2} \rho} \frac{\partial P}{\partial w}=g_{w}$,

$\frac{v}{h_{2}} \frac{\partial P}{\partial w}-\frac{\gamma}{h_{2}} \frac{P v}{\rho} \frac{\partial \rho}{\partial w}=-(\gamma-1) \Lambda$,

$\frac{v}{h_{2}} \frac{\partial P_{\mathrm{e}}}{\partial w}-\frac{\gamma}{h_{2}} \frac{P_{\mathrm{e}} v}{\rho} \frac{\partial \rho}{\partial w}=-(\gamma-1)(\Lambda-\Gamma)$,

where $C$ is a constant proportional to the mass flux onto the stellar surface, $C=\dot{m} h_{1 *} h_{3 *}$. The functions $h_{1}(u, w)$ and $h_{3}(u, w)$ are metric terms of the curvilinear coordinate system (see Appendix A). The term $g_{w}=\mathbf{g} \cdot \hat{\boldsymbol{w}}$ is gravitational acceleration along the magnetic field line (i.e. in the $w$ direction).

As expressed in Saxton et al. (2005), the rate of energy exchange from ions to electrons has the form

$\Gamma=X \rho^{5 / 2} P_{\mathrm{e}}^{-3 / 2}\left(P-\chi P_{\mathrm{e}}\right)$,

where $X$ and $\chi$ are numerical constants that depend on the plasma composition. The volumetric cooling rate of gas due to thermal bremsstrahlung radiation is approximated as

$\Lambda_{\mathrm{br}}=A \rho^{2} \sqrt{\frac{P_{\mathrm{e}}}{\rho}}$,

with $A$ being another constant depending on composition. Saxton et al. (2005) gave derivations of $A, X$ and $\chi$ and their values for a plasma of solar metallicity. We assume a total cooling function that includes bremsstrahlung and cyclotron contributions,

$\Lambda=\Lambda_{\mathrm{br}}+\Lambda_{\mathrm{cy}}=\Lambda_{\mathrm{br}}\left[1+\epsilon_{\mathrm{s}} f_{\mathrm{cy}}\left(\rho, P_{\mathrm{e}}\right)\right]$,

with a cyclotron/bremsstrahlung emissivity ratio given by the function $f_{\text {cy }}$ defined by Wu (1994); Wu et al. (1994); Saxton et al. (1997), refined by Saxton \& Wu (1999); Cropper et al. (1999); Saxton et al. (2005) and extended to the dipolar accretion model in Canalle et al. (2005).

We rewrite the flow density, total pressure and electron partial pressure in terms of the $h$ functions, the flow constant $C$ and variables that have the dimensions of velocity,

$$
\begin{aligned}
& \rho=\frac{C}{h_{1} h_{3} v} \\
& P=\frac{C}{h_{1} h_{3}}(\xi-v) \\
& P_{\mathrm{e}}=\frac{C}{h_{1} h_{3}}(\xi-v)\left(\frac{\sigma}{\sigma+1}\right),
\end{aligned}
$$


where $\xi$ measures the ratio of momentum to mass fluxes (introduced in Cropper et al. 1999) and $\sigma \equiv P_{\mathrm{e}} / P_{\mathrm{i}}$, the electron to ion partial pressure ratio. For the sake of further brevity, we define

$p \equiv \frac{h_{1} h_{3}}{C} P_{\mathrm{e}}=\frac{\sigma}{\sigma+1}(\xi-v)$.

We separate the finite and $v^{-1}$ terms in the electron-ion energy exchange function and the bremsstrahlung radiative cooling functions,

$\Gamma=X\left(\frac{C}{h_{1} h_{3}}\right)^{2} \frac{\sigma+1-\chi \sigma}{\sigma p^{1 / 2} v^{5 / 2}} \equiv \frac{C}{h_{1} h_{2} h_{3}} \hat{\Gamma} v^{-5 / 2}$,

$\Lambda_{\mathrm{br}}=A\left(\frac{C}{h_{1} h_{3}}\right)^{2} p^{1 / 2} v^{-3 / 2} \equiv \frac{C}{h_{1} h_{2} h_{3}} \tilde{\Lambda} v^{-3 / 2}$.

The functions $\hat{\Gamma} \equiv h_{1} h_{2} h_{3} \Gamma v^{5 / 2} / C$ and $\tilde{\Lambda} \equiv h_{1} h_{2} h_{3} \Lambda v^{3 / 2} / C$ lack explicit $v$ dependencies and are finite everywhere.

As in Canalle et al. (2005), the equations for momentum and total energy provide

$\frac{d \xi}{d w}=\frac{g_{w} h_{2}}{v}+\mathcal{H}(\xi-v)$

$\frac{d v}{d w}=\frac{-\left[(\gamma-1) \tilde{\Lambda} v^{-3 / 2}+\gamma \mathcal{H}(\xi-v) v+h_{2} g_{w}\right]}{\gamma(\xi-v)-v}$

Definition (14), equations (6) and (19) yield

$$
\begin{aligned}
& \frac{d P_{\mathrm{e}}}{d w}=\frac{P_{\mathrm{e}}}{\xi-v}\left(\frac{d \xi}{d w}-\frac{d v}{d w}\right)-\mathcal{H} P_{\mathrm{e}}+\frac{\left(P-P_{\mathrm{e}}\right)^{2}}{P} \frac{d \sigma}{d w}, \\
& \frac{d \sigma}{d w}=-\frac{(\gamma-1)(\sigma+1)}{(\xi-v) v^{7 / 2}}[v \tilde{\Lambda}-(\sigma+1) \hat{\Gamma}] .
\end{aligned}
$$

Strong shock conditions apply at the outer boundary, while the condition $v=0$ applies at the stellar surface $(r=1)$. Our particular method of numerical solution is described in Appendix B] We adopt the white dwarf massradius relation of Nauenberg (1972).

\section{RESULTS}

\subsection{Structure of the post-shock flow}

Table 1 summarises the basic properties of several models of $\mathrm{mCVs}$, with nearly direct (approximately radial) accretion onto a magnetic pole (colatitude $\theta_{*}=0.001^{\circ}$ ) and hotspot area $10^{15} \mathrm{~cm}^{2}$. We consider white dwarfs in the mass range $0.7-1.2 M_{\odot}$, with surface magnetic field strengths $B_{*} \leqslant 50 \mathrm{MG}$. The ratio of electron to ion temperatures at the shock, $\left(T_{\mathrm{e}} / T_{\mathrm{i}}\right)_{\mathrm{s}}$, is treated as a free parameter (e.g. as in Imamura et al. 1996; Saxton \& Wu 1999; Saxton et al. 2005). This ratio has little effect on the shock height, $R_{\mathrm{s}}$. This implies that the shock position is insensitive to the details of heating at the shock precursor, but depends mainly on the white-dwarf's properties, the inflow rate and field geometry.

Figure 1 shows how the shock radius varies with the accretion colatitude $\theta_{*}$, the magnetic field strength and accretion rate. These illustrative models assume that $T_{\mathrm{e}}=T_{\mathrm{i}}$ at the shock, and accretion hot-spot area of $10^{15} \mathrm{~cm}^{2}$. In all calculations (as in Canalle et al. 2005) the shock is closer to the stellar surface when $\dot{m}$ is greater. The greatest variation of $R_{\mathrm{s}}$ with $\theta_{*}$ occurs in systems where the shock radius is
Table 1. Parameters and properties of a set of representative accretion shocks, varying the electron to ion temperature ratio at the shock $\left(T_{\mathrm{e}, \mathrm{s}} / T_{\mathrm{i}, \mathrm{s}}\right)$. The white-dwarf surface magnetic field is given by $B_{7}=B_{*} / 10 \mathrm{MG}$, and the specific accretion rate $\dot{m}$ is in units of $\mathrm{g} \mathrm{cm}^{-2} \mathrm{~s}^{-1}$. If the magnetic field at the hot-spot is $B_{*}$ then its magnitude at the pole is $B_{\mathrm{p}}=2 B_{*} / \sqrt{4-3 u}$.

\begin{tabular}{ccccccc}
\hline \multicolumn{2}{c}{$T_{\mathrm{e}, \mathrm{s}} / T_{\mathrm{i}, \mathrm{s}}:$} & & & & 1.0 & 0.5 \\
$M_{\mathrm{w}}$ & $B_{7}$ & $\dot{m}$ & $\theta_{*}$ & $a_{15}$ & $r_{\mathrm{s}}$ & $r_{\mathrm{s}}$ \\
& & & & & & \\
\hline 0.7 & 0.0 & 1.0 & 0.0 & 1.0 & 1.081 & 1.080 \\
0.7 & 1.0 & 1.0 & 0.0 & 1.0 & 1.047 & 1.047 \\
0.7 & 3.0 & 1.0 & 0.0 & 1.0 & 1.010 & 1.011 \\
0.7 & 5.0 & 1.0 & 0.0 & 1.0 & 1.005 & 1.005 \\
& & & & & & \\
1.0 & 0.0 & 1.0 & 0.0 & 1.0 & 1.610 & 1.617 \\
1.0 & 1.0 & 1.0 & 0.0 & 1.0 & 1.096 & 1.103 \\
1.0 & 3.0 & 1.0 & 0.0 & 1.0 & 1.015 & 1.017 \\
1.0 & 5.0 & 1.0 & 0.0 & 1.0 & 1.007 & 1.008 \\
& & & & & & \\
1.2 & 1.0 & 1.0 & 0.0 & 1.0 & 1.175 & 1.206 \\
1.2 & 3.0 & 1.0 & 0.0 & 1.0 & 1.024 & 1.028 \\
1.2 & 5.0 & 1.0 & 0.0 & 1.0 & 1.011 & 1.014 \\
& & & & & & \\
\hline
\end{tabular}

large compared to $R_{\mathrm{w}}$. We find that the $1 \mathrm{~T}$ model (dotted curves) predicts lower shock positions than the $2 \mathrm{~T}$ model does. The difference is proportionally greater in large- $B_{*}$ cases, where the shock is typically nearer the stellar surface. As the $2 \mathrm{~T}$ model predicts increased $R_{\mathrm{s}}$ values, the shock temperatures are lower, $T_{\mathrm{s}} \propto R_{\mathrm{S}}^{-1}$. This effect is greatest for large $M_{\mathrm{w}}$.

Figure2 2 portrays the radial distribution of electron density $\left(n_{\mathrm{e}}\right)$ and temperature $\left(k T_{\mathrm{e}}\right)$ in accretion models with $\theta_{*}=0^{\circ}, M_{\mathrm{w}}=1.0 M_{\odot}$ and $\dot{m}=2 \mathrm{~g} \mathrm{~cm}^{-2} \mathrm{~s}^{-1}$. The $1 \mathrm{~T}$ and $2 \mathrm{~T}$ models predict similar density and thermal structures in the lower field cases $\left(B_{*}=0 \mathrm{MG}\right)$. However for stronger magnetic fields $\left(B_{*}=50 \mathrm{MG}\right)$ the models predict considerably different shock heights and qualitatively different density and temperature distributions. In $2 \mathrm{~T}$ structures, the hottest electrons are confined to a narrower region near the shock than in $1 \mathrm{~T}$ shocks with equivalent system parameters. Plotting the $T_{\mathrm{e}}$ versus $n_{\mathrm{e}}$ distributions, as in the lower panels of Figure 2 shows that in $2 \mathrm{~T}$ models with strong magnetic fields the density of the hottest gas is lower than in $1 \mathrm{~T}$ models. This will have consequences for the post-shock X-ray emissions.

\subsection{X-ray emissions}

For each of the solutions for the post-shock accretion flow, we calculate an X-ray spectrum following the methods of Cropper et al. (1999) and Saxton et al. (2005). The postshock volume is divided into $\gtrsim 10^{4}$ strata according to the spatial steps of the numerically integrated flow profile. The volume of each stratum is inferred from the step size $d w$, the hot-spot area and the crossectional area scaling factor $h_{1} h_{3} / h_{1 *} h_{3 *}$. The local electron density and temperature $\left(n_{\mathrm{e}}, T_{\mathrm{e}}\right)$ are obtained from the dimensionless flow model and the scaling units, $\left(R_{\mathrm{w}}, V_{\mathrm{w}}, C\right)$. We assume that the $\mathrm{X}$ ray line and continuum emission are optically thin, and in- 

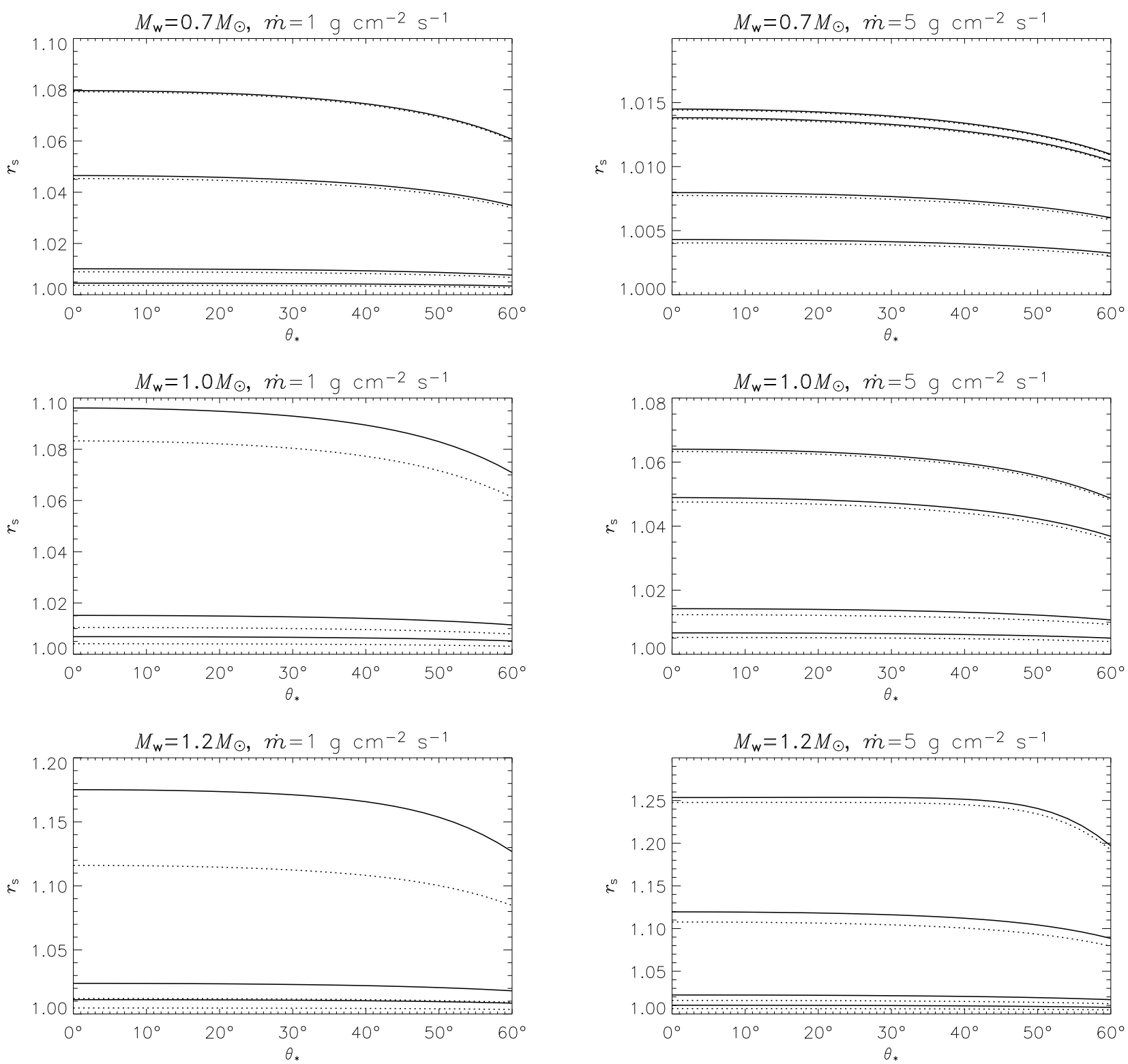

Figure 1. Effect of the accretion colatitude $\left(\theta_{*}\right)$ upon the shock radius, $r_{\mathrm{s}} \equiv R_{\mathrm{S}} / R_{\mathrm{W}}$ in units of the stellar radius, for cases with different specific accretion rates $(\dot{m})$, white-dwarf mass $\left(M_{\mathrm{w}}\right)$ and magnetic field $\left(B_{*}\right)$. The accretion hot-spot area is $10^{15} \mathrm{~cm}^{2}$. Solid lines mark the results of the $2 \mathrm{~T}$ model; dotted lines denote the corresponding results of the $1 \mathrm{~T}$ model. Within each panel, paired curves from bottom to top represent cases with field strengths $B_{*}=50,30,10 \mathrm{MG}$; a fourth pair shows cases with neglible cyclotron cooling (yet channelled by a dipole funnel). These low-field cases are insoluble when $\dot{m}=1 \mathrm{~g} \mathrm{~cm}^{-2} \mathrm{~s}^{-1}$. and $M_{\mathrm{w}}=1.0,1.2 M_{\odot}$.

voke the XSPEC implementation of the MEKAL thermal plasma model (Mewe et al. 1985; Kaastra \& Mewe 1993) to calculate emissivities for local $\left(n_{\mathrm{e}}, T_{\mathrm{e}}\right)$ values. Volumetric integration over all the post-shock strata yields a synthetic X-ray spectrum. We exclude the densest strata, with $n_{\mathrm{e}} \geqslant 10^{18} \mathrm{~cm}^{-3}$, which the spectral model cannot handle (and which in any case represent optically thick layers merging into the atmosphere of the white dwarf). We omit the effect of shadowing by a white dwarf which obscures its own accretion inflow.

Saxton et al. (2005) studied 2T effects in a planar accretion model, without the effects of gravity and varying width of the magnetic accretion funnel. They found that $2 \mathrm{~T}$ effects change the keV X-ray spectra most significantly for cases with larger $M_{\mathrm{w}}$. For given system parameters, the $2 \mathrm{~T}$ model gave a harder continuum but relatively weaker line emission. The ratio of electron to ion temperatures at the shock was found to have little effect on the spectra. This was explained in terms of the concentration of X-ray emission near the stellar surface (well downstream from the shock) by which point collisional energy exchanges have nearly equilibrated the electrons and ions.

Our present calculations, with a dipolar accretion funnel and $2 \mathrm{~T}$ effects, typically produce spectra such as those in Figure 3 The slope of the continuum is steep, but there are variations in the curvature of the continuum and the details 

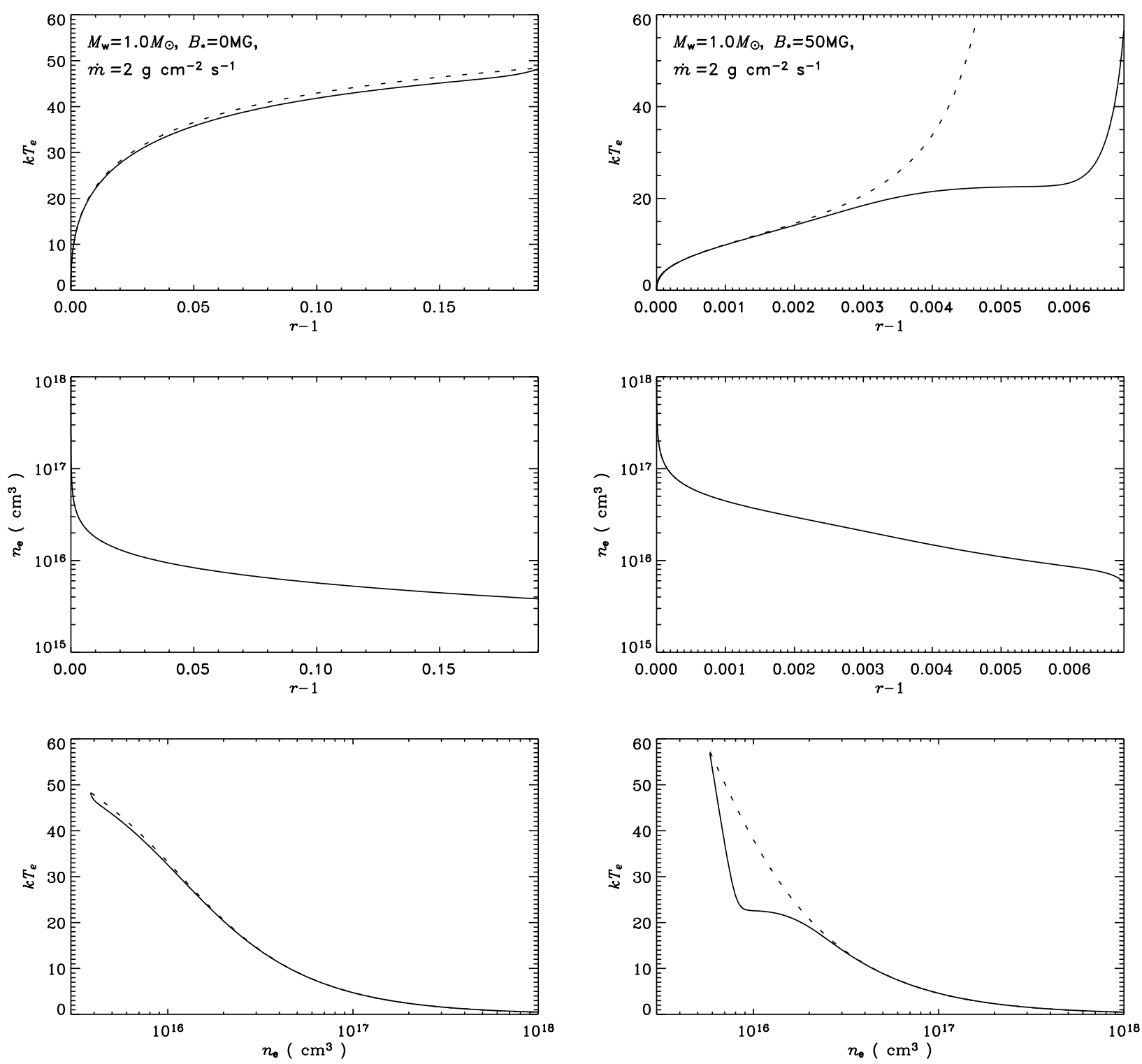

Figure 2. Profiles of electron temperature (upper row) and electron number density (middle row) for accretion onto $1.0 M_{\odot}$ white dwarfs, as a function of height above the surface. The left and right columns respectively show cases with negligible cyclotron cooling, and with dominant cyclotron cooling. The accretion spot is at the pole $\left(\theta_{*}=0^{\circ}\right)$. Solid lines mark the results of the $2 \mathrm{~T}$ model; dotted lines denote the results of the $1 \mathrm{~T}$ model. Electron and ion temperatures match at the shock and stellar surface, but the electrons are cooler elsewhere. The bottom row shows the temperature/density phase structure: in high-field cases the $2 \mathrm{~T}$ model has lower densities in the hotter strata.

of line strengths. The effects of the various system parameters are best illustrated by plots of the ratios of spectra calculated with different conditions or assumptions.

\subsubsection{Dipolar vs planar accretion}

Figure 4 shows the ratios of $\mathrm{X}$-ray spectra calculated in $2 \mathrm{~T}$ models with identical system parameters but dipolar verses planar accretion geometry. In all the cases we calculated, the dipolar model provides a harder continuum, and reduced line emission, particularly for photon energies $\lesssim 1 \mathrm{keV}$. These effects are small for a white dwarf of $0.7 M_{\odot}$, but are more considerable for $1.0 M_{\odot}$ and more massive cases. The dipolar $(2 \mathrm{Td})$ and planar $(2 \mathrm{Tp})$ models are more in agreement regarding continuum at photon energies $\lesssim 1 \mathrm{keV}$ if the accretion hot-spot is at high $\theta_{*}$ (further from the magnetic pole).

\subsubsection{Two-temperature vs one-temperature dipolar inflows}

Figure 5 shows the quotients of X-ray spectra over the $0.2-10 \mathrm{keV}$ band, from $1 \mathrm{~T}$ and $2 \mathrm{~T}$ dipolar calculations for a white-dwarf mass of $0.7 M_{\odot}$. The X-ray continuum is generally brighter in a $2 \mathrm{~T}$ model. In cases where bremsstrahlung 
is the dominant cooling process $\left(B_{*}=0 \mathrm{MG}\right)$ the X-ray continuum has equivalent spectral slopes in $1 \mathrm{Td}$ and $2 \mathrm{Td}$ models, up to photon energies $\sim 5 \mathrm{keV}$. Above this energy, the $1 \mathrm{Td}$ model predicts a marginally harder continuum. The $1 \mathrm{Td}$ models predict $\sim 0.5 \%$ stronger emission lines around $1 \mathrm{keV}$, but lines around $\sim 7 \mathrm{keV}$ are weakened by a similar degree.

For non-zero but small magnetic fields $\left(B_{*}=10 \mathrm{MG}\right)$ the shape of the continuum is similar to the zero-field cases, but the $1 \mathrm{Td}$ and $2 \mathrm{Td}$ calculations yield greater discrepancies about the line strengths, up to $\sim 1 \%$. The specific accretion rate, $\dot{m}$, has a slightly greater effect on the disagreements between the predicted lines.

For a stronger magnetic field $\left(B_{*}=30 \mathrm{MG}\right)$ the $1 \mathrm{Td}$ model yields a softer continuum than equivalent $2 \mathrm{Td}$ calculations. The $1 \mathrm{Td}$ model generally predicts stronger emission lines, by up to $\sim 2 \%$ for the cases with lowest $\dot{m}$. Two-temperature effects are less apparent in the keV X-ray continuum and lines when the accretion rate is larger. The spectral effects generally strengthen as the magnetic field increases; the greatest extreme in our plots is the case with $B_{*}=50 \mathrm{MG}$ and $\dot{m}=1 \mathrm{~g} \mathrm{~cm}^{-2} \mathrm{~s}^{-1}$.

The discrepancy between spectra predicted in the $1 \mathrm{Td}$ and $2 \mathrm{Td}$ models is more acute for systems with larger whitedwarf mass. For cases with $M_{\mathrm{w}}=1.0 M_{\odot}$ ( oversoftness of the $1 \mathrm{Td}$ predicted continuum is more pronounced in the $B_{*}=30 \mathrm{MG}$ case than in the comparable $B_{*}=50 \mathrm{MG}$ cases with $M_{\mathrm{w}}=0.7 M_{\odot}$. The $1 \mathrm{Td} / 2 \mathrm{Td}$ pattern of over- and under-prediction of line emission between $\sim 7 \mathrm{keV}$ and $\sim 9 \mathrm{keV}$ varies considerably with $B_{*}$ and $\dot{m}$ (see the upper right panels of Figure 6).

For systems with a white dwarf with $M_{\mathrm{w}}=1.2 M_{\odot}$, the discrepancies are even greater than for $M_{\mathrm{w}}=1.0 M_{\odot}$ cases: the continuum around $\sim 10 \mathrm{keV}$ can be around $10 \%$ underpredicted in the $1 \mathrm{Td}$ model, relative to the $2 \mathrm{Td}$ calculation (Figure 7). A downward spike in the $\mathrm{H}$-like $\mathrm{Fe} \mathrm{K} \alpha$ emission that appears for $1.0 M_{\odot}$ cases (in Figure 6 representing underprediction of this line by the $1 \mathrm{Td}$ model) is absent when $M_{\mathrm{w}}=1.2 M_{\odot}$, with all other parameters equal.

\subsubsection{Latitude dependence}

The X-ray spectrum also varies with the location of the accretion spot on the white-dwarf surface. Latitudinal effects are slight when the shock height is small compared to the stellar radius (e.g. $M_{\mathrm{w}}=0.7 M_{\odot}, \dot{m}=5 \mathrm{~g} \mathrm{~cm}^{-2} \mathrm{~s}^{-1}$ ); then we obtain $\lesssim 2 \%$ enhancements in the $\sim 1 \mathrm{keV}$ lines. For the same white-dwarf mass but lower accretion rate (e.g. $M_{\mathrm{w}}=0.7 M_{\odot}, \dot{m}=1 \mathrm{~g} \mathrm{~cm}^{-2} \mathrm{~s}^{-1}$ ), a low-latitude accretion stream provides a softer X-ray continuum than polar accretion. Figure 8 shows the ratios of spectra: for inclined accretion cases $\left(\theta_{*}=10^{\circ}, 30^{\circ}\right.$ and $\left.60^{\circ}\right)$ compared to vertical accretion onto the pole $\left(\theta_{*}=0^{\circ}\right)$. For low-latitude accretion, lines are enhanced up to $\lesssim 20 \%$ at photon energies $\lesssim 1 \mathrm{keV}$. In the cases with $M_{\mathrm{w}}=0.7 M_{\odot}$, the size of $\theta_{*}$-dependency appears comparable for systems with low and high magnetic field strengths.

However in systems with a more massive white dwarf, $M_{\mathrm{w}}=1.2 M_{\odot}$, the emission depends more sensitively on $\theta_{*}$, for any field strength $B_{*}$. In the cases with the shock at a relatively low radius (e.g. $M_{\mathrm{w}}=1.2 M_{\odot}, \dot{m}=5 \mathrm{~g} \mathrm{~cm}^{-2} \mathrm{~s}^{-1}$ ) the spectra are almost indistinguishable for $\theta_{*}=0^{\circ}, 10^{\circ}$; the $\theta_{*}=30^{\circ}$ cases differ from $\theta_{*}=0^{\circ}$ by $\lesssim 1 \%$ in the $\sim 1 \mathrm{keV}$ lines. At $\theta_{*}=60^{\circ}$ the enhancement of line emission is $\lesssim 7 \%$ when $B_{*}=10 \mathrm{MG}$, but half as great when $B_{*}=$ 50 MG. A similar variation with $B_{*}$ occurs among cases with $M_{\mathrm{w}}=1.2 M_{\odot}$ and $\dot{m}=1 \mathrm{~g} \mathrm{~cm}^{-3} \mathrm{~s}^{-1}$ — the greatest $\theta_{*}$ dependency appears in the systems with the greatest relative shock height, $r_{\mathrm{s}}$.

\section{DISCUSSION}

\subsection{Summary for magnetic cataclysmic variables}

The incorporation of $2 \mathrm{~T}$ effects in the post-shock accretion flows of mCVs generally hardens the emitted keV X-ray continuum. A dipolar accretion funnel further hardens the spectrum of a $2 \mathrm{~T}$ flow, relative to models with simpler planar geometry. A 2T dipolar model predicts a harder spectrum than a $2 \mathrm{~T}$ planar model, if the white dwarf is as massive as $1 M_{\odot}$ or more. The differences between planar and dipolar $2 \mathrm{~T}$ accretion models are greatest in systems where the shock height is large compared to the stellar radius: i.e. greater $M_{\mathrm{w}}$ or lower $B_{*}$. The spectral predictions of $1 \mathrm{~T}$ and $2 \mathrm{~T}$ dipolar models differ more significantly when the white dwarf is massive, has a strong magnetic field or low specific accretion rate. Line emission is typically weaker around $\sim 1 \mathrm{keV}$ and $\sim 7-10 \mathrm{keV}$ in a $2 \mathrm{~T}$ flow than in a $1 \mathrm{~T}$ flow, except for the Fe $\mathrm{K} \alpha$ lines, which could become stronger.

This is qualitatively consistent with the $2 \mathrm{~T}$ effects found for basic planar accretion models without the inclusion of gravity in the hydrodynamic equations (Saxton et al. 2005); Two-temperature effects upon X-ray spectra persist despite the introduction of a dipolar magnetic field geometry, which in the $1 \mathrm{~T}$ model of Canalle et al. (2005) soften the X-ray spectra relative to the results of Cropper et al. (1999). This softening due to funnel geometry is, however, smaller than the hardening apparent when $2 \mathrm{~T}$ physics is incorporated. The 2Td model predicts the most hard photons for a given mass $M_{\mathrm{w}}$, and therefore its spectral fit to an observed spectrum will require a lower mass than the standard planar and $1 \mathrm{~T}$ models do.

\subsection{Inferred masses}

To quantify how the mass estimates are affected, we calculated spectra for models with different $M_{\mathrm{w}}$, with the other parameters fixed $\left(\theta_{*}=0^{\circ}, \dot{m}=2 \mathrm{~g} \mathrm{~cm}^{-2} \mathrm{~s}^{-1}\right)$, for medium $\left(B_{*}=20 \mathrm{MG}\right)$ and strong-field $\left(B_{*}=50 \mathrm{MG}\right)$ cases. For a given white dwarf mass, we manually match the shape of the X-ray continuum by adjusting the masses in $1 \mathrm{Tp}$, 1Td, 2Tp and 2Td models (Wu 1994; Canalle et al. 2005; Saxton et al. 2005, this paper respectively). Each fit is defined by matching the ratio of spectra at $0.1 \mathrm{keV}$ and $10 \mathrm{keV}$, with the flattest possible ratio at intermediate energies. Figure9 illustrates how the $2 \mathrm{~T}$ and dipolar funnel effects change spectral inferences of the white dwarf mass $\left(M_{\mathrm{w}}\right)$ from Xray observations. For low-mass white dwarfs $\left(M_{\mathrm{w}} \approx 0.5 M_{\odot}\right)$ the $1 \mathrm{~T}$ and $2 \mathrm{~T}$ spectral predictions are similar. However for cases with higher mass, say $M_{\mathrm{w}} \gtrsim 1.0 M_{\odot}$, the spectra differ considerably (middle panels).

For large $M_{\mathrm{w}}$, the $2 \mathrm{Tp}$ models require lower masses than $1 \mathrm{Tp}$ models. For $B_{*}=50 \mathrm{MG}$ the $1 \mathrm{Td}$ model requires 

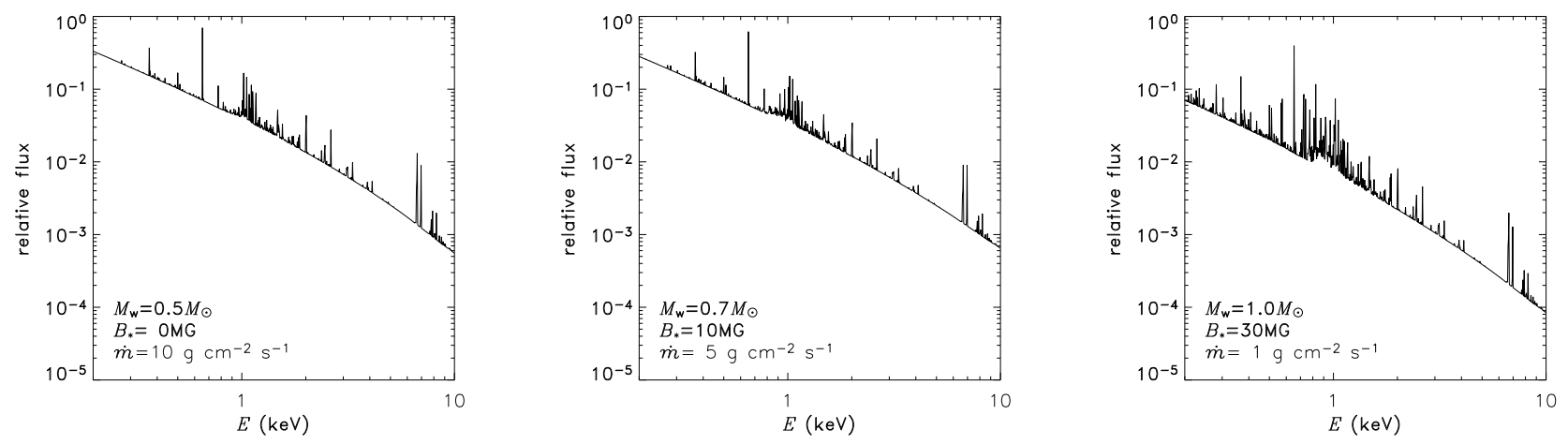

Figure 3. X-ray spectra for photon energies $0.2-10 \mathrm{keV}$, calculated for dipole-field channelled $2 \mathrm{~T}$ accretion onto white dwarfs with parameters as indicated in the panels.
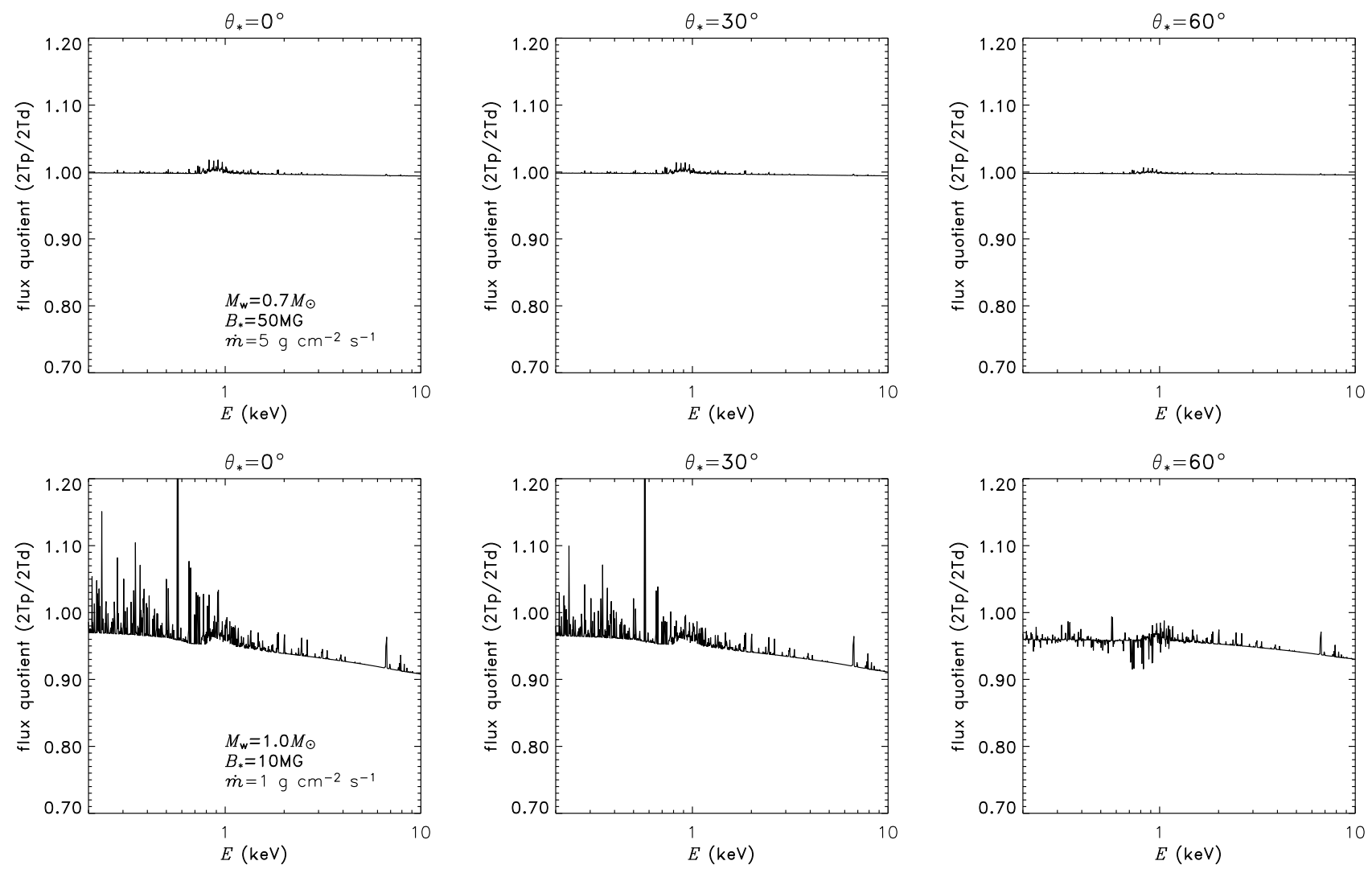

Figure 4. Quotients of spectra in $2 \mathrm{~T}$ planar accretion model to spectra in the corresponding dipolar accretion model $(2 \mathrm{Tp} / 2 \mathrm{Td})$. The columns show different cases of the accretion colatitude, $\theta_{*}=0^{\circ}, 30^{\circ}, 60^{\circ}$. The top row represents a system in which the shock height is small compared to the stellar radius. The bottom row shows a system with a relatively high shock. In the latter, spectra differ significantly between the dipolar and planar models.

greater masses than $2 \mathrm{Tp}$ models; but for $B_{*}=20 \mathrm{MG}$ the $1 \mathrm{Tp}$ model is more massive. The $2 \mathrm{~T}$ dipolar models consistently need the lowest $M_{\mathrm{w}}$ (see corresponding fitted masses in Figure 10). The introduction of dipolar geometry to 2temperature flows (from $2 \mathrm{Tp}$ to $2 \mathrm{Td}$ models) has hardened the spectra. For example, a $1.00 M_{\odot} 2 \mathrm{Tp}$ model matches a 2 Td model with $M_{\mathrm{w}} \approx 0.98 M_{\odot}$ (i.e. a $2 \%$ discrepancy, see Table 2). The X-ray continuum of a $1.00 M_{\odot} 1 \mathrm{Td}$ model is best matches a $2 \mathrm{Td}$ model with $M_{w} \approx 0.95 M_{\odot}$ (a $5 \%$ discrepancy, see Figure 9, lower middle panel).

We note that the flow geometry and $2 \mathrm{~T}$ conditions can affect the line spectra as well as the continuum. Here, we demonstrate that mass estimates obtained from the continuum fitting depends on the assumed geometry of the flow. If the spectral line information were also considered, we would need to consider variations of the values of $B_{*}, \dot{m}, \theta_{*}$ and 

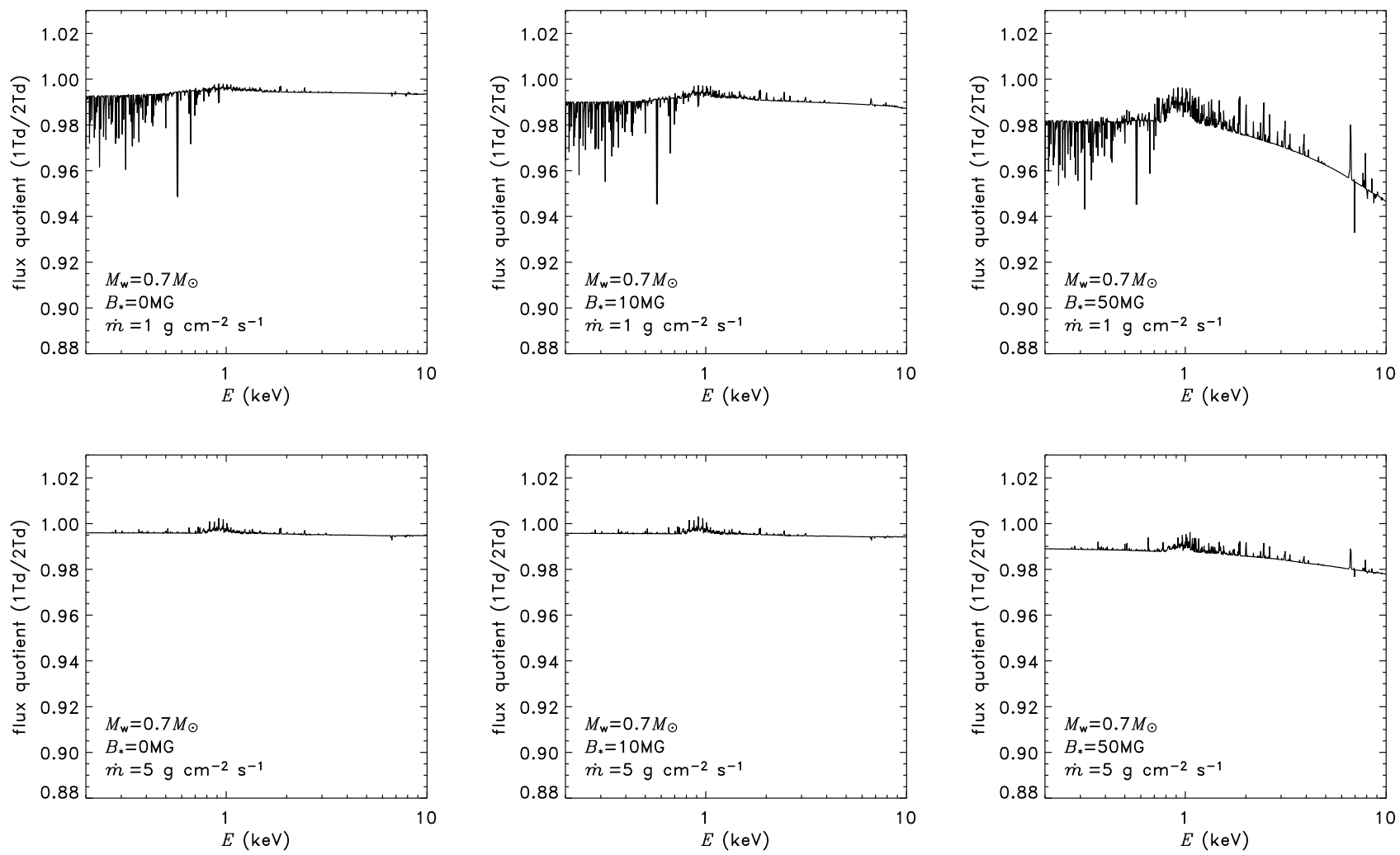

Figure 5. Quotients of spectra in $1 \mathrm{~T}$ and $2 \mathrm{~T}$ dipolar models $(1 \mathrm{Td} / 2 \mathrm{Td})$. In these cases the white-dwarf mass is $0.7 M_{\odot}$ and accretion occurs onto the pole $\left(\theta_{*}=0^{\circ}\right)$.

the hot-spot area too. For the present study, it suffices to show that the joint inclusion of $2 \mathrm{~T}$ phenomena and dipolar geometry can reduce mass estimates by $\lesssim 9 \%$ near the Chandrasekhar limit, by $\lesssim 7 \%$ near a solar mass, but only $\lesssim 1 \%$ near $0.5 M_{\odot}$.

We now consider the mass estimates of Ramsav (2000) who determined the mass of the white dwarf in $21 \mathrm{mCVs}$ - 8 polars and 13 intermediate polars (IPs) - using RXTE PCA (2-60 keV) data. Using the 1 Tp model prescribed by Cropper et al. (1999), Ramsav (2000) found a mean mass of $0.87 M_{\odot}$ and $0.80 M_{\odot}$ for his sample of IPs and polars respectively. Applying a correction factor based on our results above gives a mean mass of $0.82 M_{\odot}$ and $0.76 M_{\odot}$ for the IPs and polars respectively.

Other affects maybe important as well. For instance, Suleimanov et al. (2005) found that when they determined the mass of 14 IPs using RXTE PCA plus HEXTE (10$200 \mathrm{keV}$ ) data the mean mass of the IPs was reduced by $0.20 M_{\odot}$ to $0.75 M_{\odot}$. This was explained by the fact that in IPs broad accretion curtains with high total absorption columns are thought to be present, which can affect the modelling of the continuum even at relatively hard X-ray energies. Because polars have higher magnetic field strengths and hence more collimated accretion streams, absorption may affect their X-ray continua less than for IPs.

We note that the mean mass of white dwarfs in mCVs as determined by Suleimanov et al. (2005) and in this paper is greater than that of isolated white dwarfs $\left(0.56 M_{\odot}\right.$, Bergeron et al. 1992). We note that Wickramasinghe \& Ferrario (2005) found that white dwarfs with high magnetic fields have a mean mass of $0.93 M_{\odot}-$ significantly higher than that in isolated white dwarfs. Our mass estimates remain consistent with the scenario in which magnetic white dwarfs are more massive than their nonmagnetic counterparts.

\subsection{Further applications and scope}

Our 2-temperature formulation with magnetic funnel geometry is applicable to a variety of accreting stellar systems other than the cataclysmic variables. With substitution of the relevant radiation processes, the model is applicable to accretion onto neutron stars, and similarly it is applicable to field-aligned accretion onto $\mathrm{T}$ Tauri stars. The key assumptions are that the flow be hydrodynamic (collisional), field-aligned, and that radiation effects can be described in terms of local variables.

Our hydrodynamic formulation treats accretion flows that are steady and smooth: rapid temporal variability is omitted. Continuous flows onto some mCVs oscillate at $\sim 1 \mathrm{~Hz}$, interpreted as a thermal instability of the shock, (see Langer et al. 1981, 1982; Chevalier \& Imamura 1982; Saxton et al. 1998). This is observed optically in only a minority of systems (e.g. Middleditch 1982; Cropper et al. 1986; Imamura \& Steiman-Cameron 1986; Larsson 1987, 1989; Ramsever et al. 1993; Middleditch et al. 1997) but not 

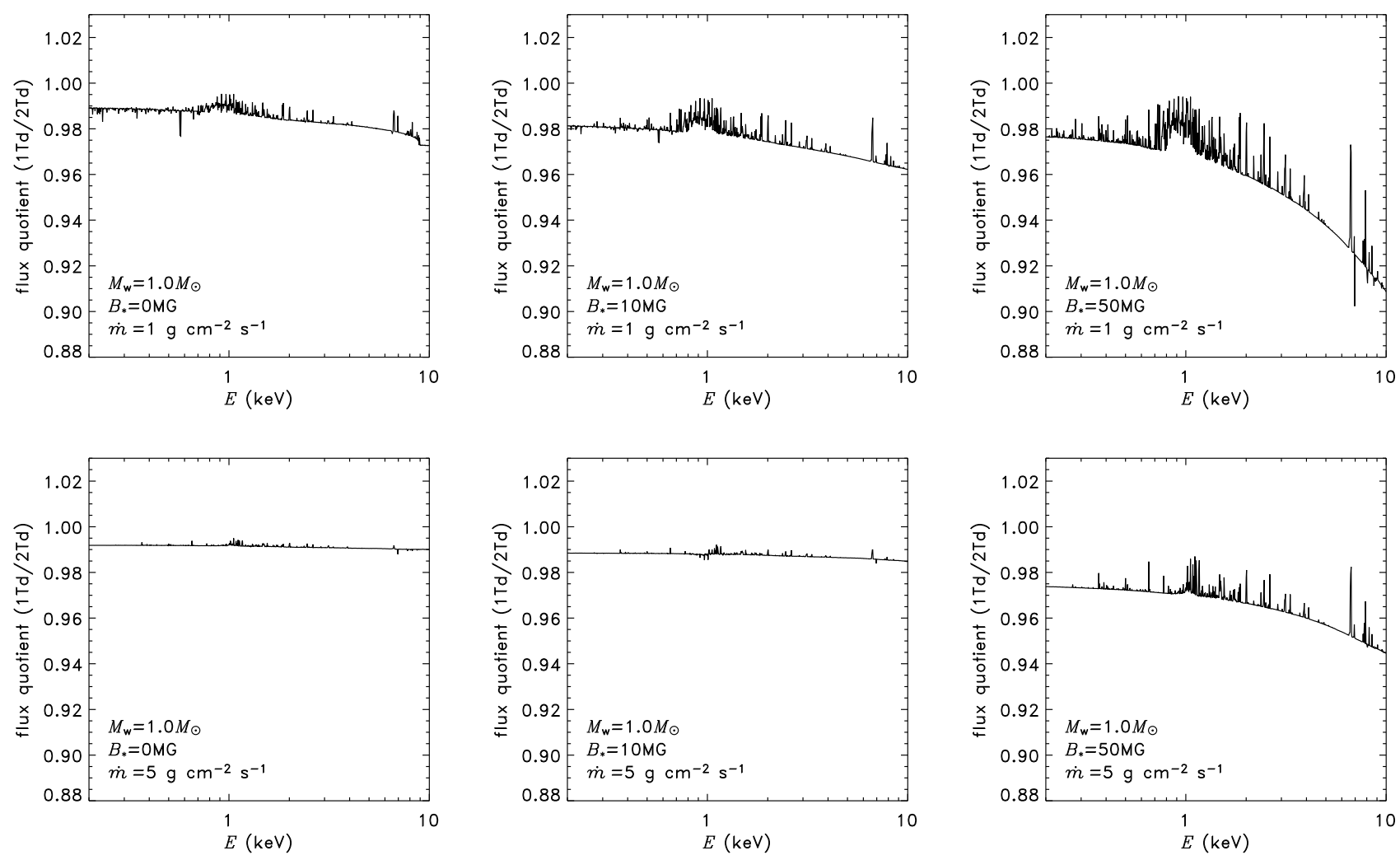

Figure 6. Quotients of spectra in $1 \mathrm{~T}$ and $2 \mathrm{~T}$ models $(1 \mathrm{Td} / 2 \mathrm{Td})$, as in Figure 5 but with $M_{\mathrm{w}}=1.0 M_{\odot}$.

yet in X-rays (e.g. Wolff et al. 1999; Christian 2000). Thus fast oscillations may not affect X-ray spectra at presently observable levels. The absence of a steady structure would preclude collisional ionisation equilibrium, which affects the spectral line strengths, as they critically depend on the density structure (see e.g. Fujimoto \& Ishida 1997; Wu et al. 2001). Our model is more applicable to cases with slower temporal variability - flaring which exceeds the post-shock free-fall time - by calculating sequences of steady models with different $\dot{m}$ values but identical $M_{\mathrm{w}}, B_{*}$ and $\theta_{*}$.

The spectral effects of spatial variations are harder to assess. Mild inhomogeneities might be modelled by treating the accretion column as a distribution of streams with differing $\dot{m}$ and cross-sections, then summing their spectra. By construction, we cannot model highly inhomogeneous flows: these would require highly resolved, time-dependent hydrodynamic simulations. In some asynchronous accreting systems, a continuous flow may fragment into dense blobs due to a magnetic drag (e.g. Arons \& Lea 1980; Frank et al. 1988; King 1993; Wynn \& King 1995). However if the magnetic field is strong enough then the blobs shred into a continuous field-aligned flow as they near to the stellar surface (Arons \& Lea 1980). If the field is weak, blobs may survive to strike the stellar surface individually. Such a shower is qualitatively different from a pressure-supported stand-off shock. However, emission from steady accretion shocks is universally observed in $\mathrm{mCVs}$ and is the major component, except at the softest energies where the emission from ballistic blobs contributes in most cases. Spectral modelling of predominantly clumpy accretion is a challenge beyond the scope of this paper.

\section{ACKNOWLEDGMENTS}

KW thanks TIARA for their hospitality during his visit there and for support through a Visiting Fellowship. TIARA is operated under Academia Sinica and the National Science Council Excellence Projects programs in Taiwan administered through grant number NSC 94-2752-M-007-001.

\section{REFERENCES}

Aizu K., 1973, Prog. Theor. Phys, 49, 1184

Arfken G. B., Weger H. J., 2001, Mathematical Methods for Physicists. Academic Press, New York

Arons J., 1993, ApJ, 408, 160

Arons J., Lea S. M., 1980, ApJ , 235, 1016

Bergeron P., Saffer R. A., Liebert J., 1992, ApJ, 394, 228

Canalle J. B. G., Saxton C. J., Wu K., Cropper M., Ramsay G., 2005, A\&A, 440, 185

Chevalier R. A., Imamura J. N., 1982, ApJ, 261, 543

Christian D. J., 2000, AJ, 119, 1930

Cropper M., 1990, Space Sci. Rev., 54, 195

Cropper M., Menzies J. W., Tapia S., 1986, MNRAS , 218, 201

Cropper M., Wu K., Ramsay G., Kocabiyik A., 1999, MNRAS, 306, 684 

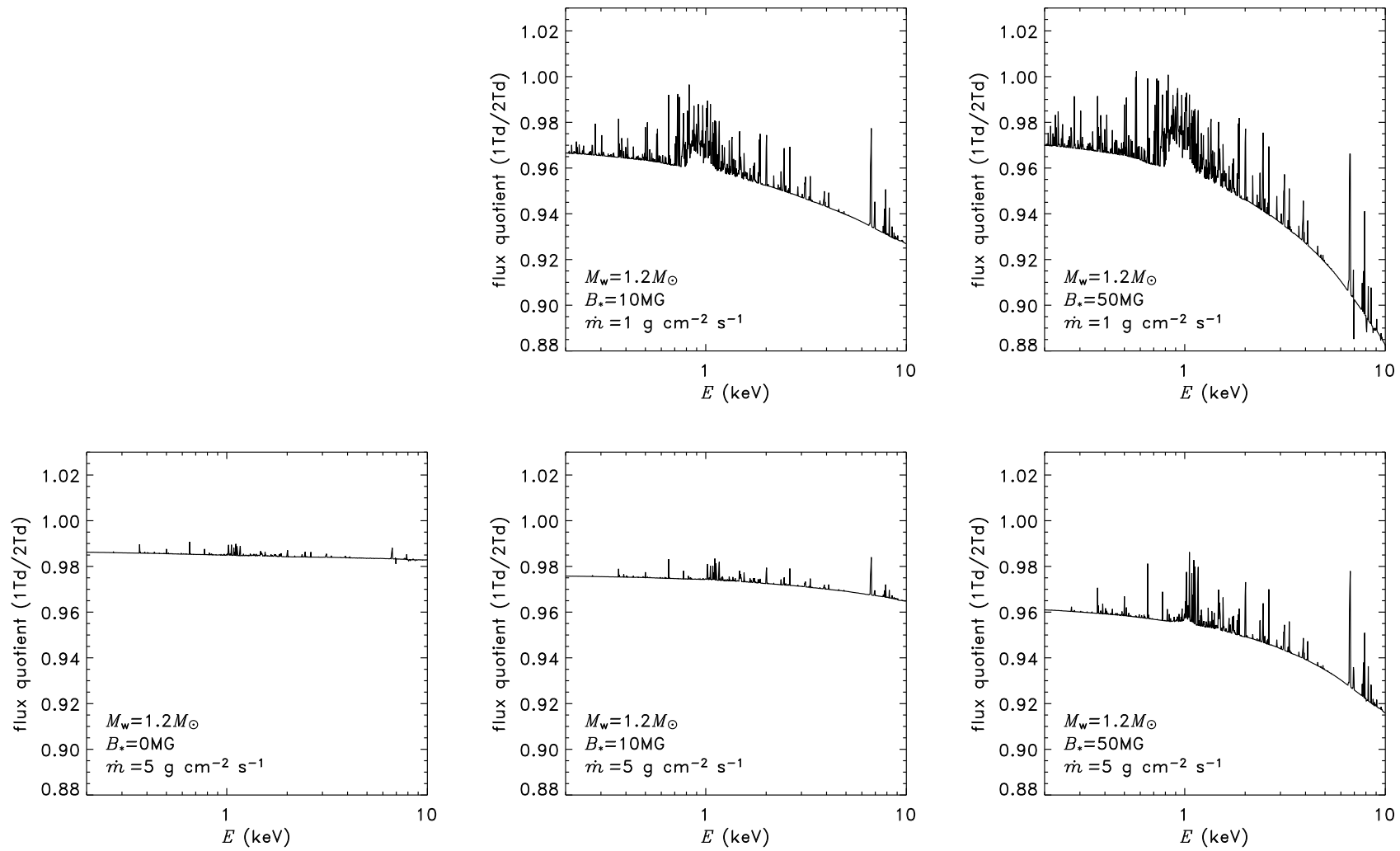

Figure 7. Quotients of spectra in $1 \mathrm{~T}$ and $2 \mathrm{~T}$ models $(1 \mathrm{Td} / 2 \mathrm{Td})$, as in Figure 5 but with $M_{\mathrm{w}}=1.2 M_{\odot}$.

Elsner R. F., Lamb F. K., 1977, ApJ, 215, 897

Fabian A. C., Pringle J. E., Rees M. J., 1976, MNRAS, 175,43

Ferrario L., 1996, PASA, 13, 87

Fischer A., Beuermann K., 2001, A\&A, 373, 211

Frank J., King A. R., Lasota J.-P., 1988, A\&A, 193, 113

Fujimoto R., Ishida M., 1997, ApJ, 474, 774

Ghosh P., Lamb F. K., 1978, ApJ, 223, L83

Gregory S. G., Jardine M., Simpson I., Donati J.-F., 2006, MNRAS, 371, 999

Gullbring E., Calvet N., Muzerolle J., Hartmann L., 2000, ApJ, 544, 927

Hartman L., Hewett R., Calvet N., 1994, ApJ, 426, 669

Heerlein C., Horne K., Schwope A. D., 1999, MNRAS , 304, 145

Imamura J. N., 1981, PhD thesis, Indiana University, U.S.A.

Imamura J. N., Aboasha A., Wolff M. T., Kent K. S., 1996, ApJ, 458, 327

Imamura J. N., Durisen R. H., Lamb D. Q., Weast G. J., 1987, ApJ, 313, 298

Imamura J. N., Steiman-Cameron T. Y., 1986, ApJ , 311, 786

Kaastra J. S., Mewe R., 1993, A\&AS, 97, 443

King A. R., 1993, MNRAS, 261, 144

King A. R., Lasota J. P., 1979, MNRAS , 188, 653

Koldoba A. V., Lovelace R. V. E., Ustyugova G. V., Romanova M. M., 2002, AJ, 123, 2019

Königl A., 1991, ApJ, 370, L39
Kryukov I. A., Pogorelov N. V., Bisnovatyi-Kogan G. S., Anzer U., Börner G., 2000, A\&A, 364, 901

Lamb D. Q., Masters A. R., 1979, ApJ, 234, L117

Lamzin S. A., Stempels H. C., Piskunov N. E., 2001, A\&A, 369, 965

Langer S. H., Chanmugam G., Shaviv G., 1981, ApJ, 245, L23

Langer S. H., Chanmugam G., Shaviv G., 1982, ApJ, 258, 289

Larsson S., 1987, A\&A, 181, L15

Larsson S., 1989, A\&A, 217, 146

Li J., Wickramasinghe D. T., Rüdiger G., 1996, ApJ, 469, 765

Li J., Wilson G., 1999, ApJ , 527, 910

Lovelace R. V. E., Romanova M. M., Bisnovati-Kogan G. S., 1995, MNRAS, 275, 244

Mewe R., Gronenschild E. H. B. M., van den Oord G. H. J., 1985, A\&AS, 62, 197

Middleditch J., 1982, ApJ , 257, L71

Middleditch J., Imamura J. N., Steiman-Cameron T. Y., 1997, ApJ , 489, 912

Nauenberg M., 1972, ApJ, 175, 417

Ramsay G., 2000, MNRAS, 314, 403

Ramsay G., Cropper M., Hellier C., Wu K., 1998, MNRAS, 297, 1269

Ramseyer T. F., Robinson E. L., Zhang E., Wood J. H., Stiening R. F., 1993, MNRAS , 260, 209

Romanova M. M., Ustyugova G. V., Koldoba A. V., Wick J. V., Lovelace R. V. E., 2003, ApJ, 595, 1009 

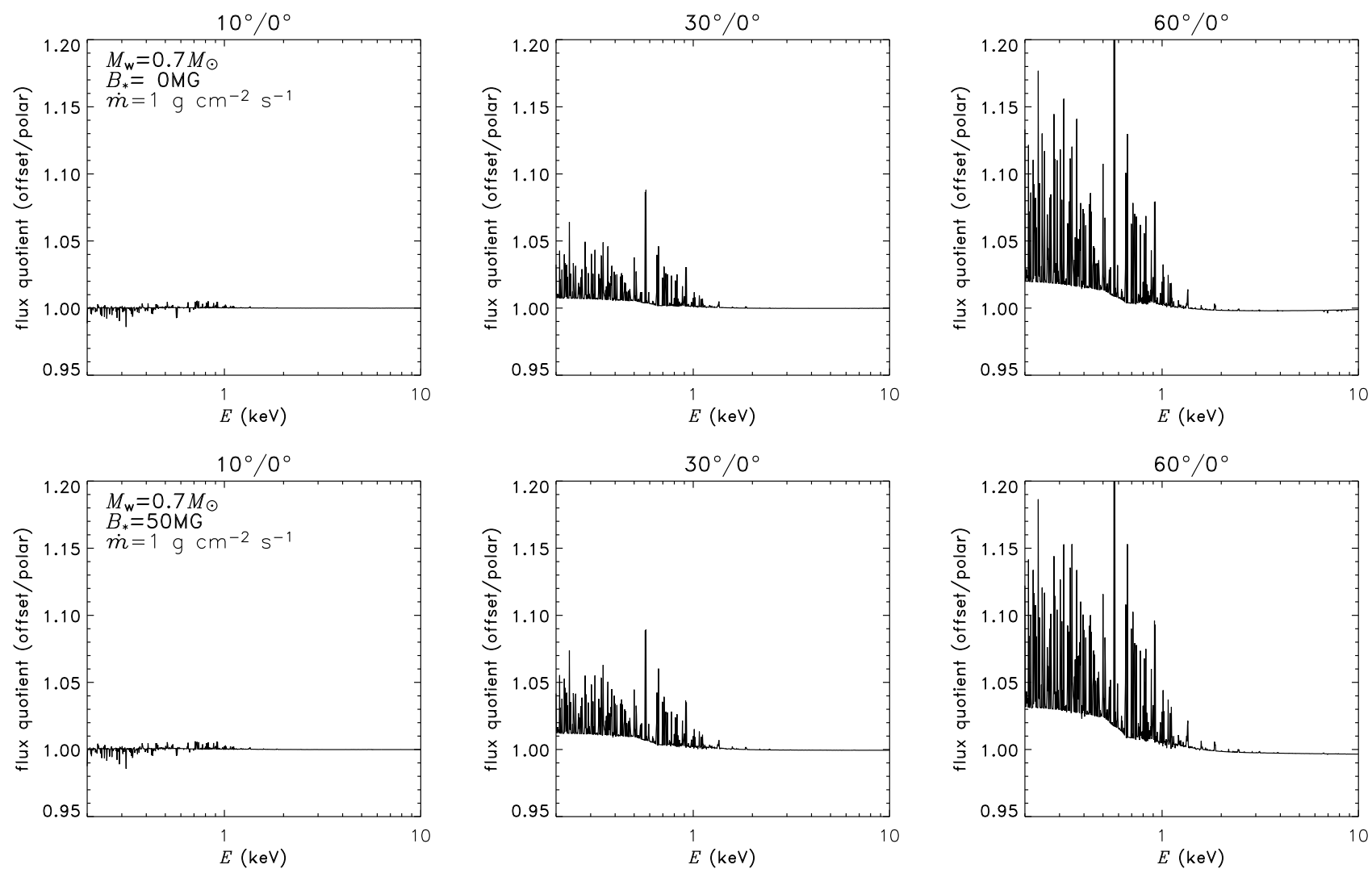

Figure 8. The effect of accretion latitude. Quotients of spectra show different colatitudes of the accretion hot-spot: $10^{\circ} / 0^{\circ}, 30^{\circ} / 0^{\circ}$ and $60^{\circ} / 0^{\circ}$ for left, middle and right columns respectively. The upper panels show cases with weaker magnetic fields; the lower panels show cases with stronger fields. In these cases the white-dwarf mass is $0.7 M_{\odot}$ and the specific accretion rate is $\dot{m}=1 \mathrm{~g} \mathrm{~cm}^{-2} \mathrm{~s}^{-1}$. All else being equal, accretion further from the pole enhances the soft X-ray continuum and lines.

Rybicki G. B., Lightman A. P., 1979, Radiative Processes in Astrophysics. Wiley, New York

Saxton C. J., Wu K., 1999, MNRAS, 310, 677

Saxton C. J., Wu K., 2001, MNRAS, 324, 659

Saxton C. J., Wu K., Cropper M., Ramsay G., 2005, MNRAS, 360, 1091

Saxton C. J., Wu K., Pongracic H., 1997, PASA, 14, 164

Saxton C. J., Wu K., Pongracic H., Shaviv G., 1998, MNRAS , 299, 862

Spitzer L., 1962, Physics of Fully Ionized Gases, 2nd Ed.. Interscience, New York

Stelzer B., Schmitt J. H. M. M., 2004, A\&A, 418, 687

Suleimanov V., Revnivtsev M., Ritter H., 2005, A\&A, 435, 191

Toropina O. D., Romanova M. M., Toropin Y. M., Lovelace R. V. E., 2003, ApJ, 593, 472

Warner B., 1995, Cataclysmic Variable Stars. Cambridge University Press, Cambridge

Wickramasinghe D. T., Ferrario L., 2005, MNRAS, 356, 1576

Woelk U., Beuermann K., 1996, A\&A, 306, 232

Wolff M. T., Imamura J. N., Middleditch J., Wood K. S., Steiman-Cameron T., 1999, in Hellier C., Mukai K., eds, ASP Conf. Ser. 157 p. 149

Wu K., 1994, PASA, 11, 61

Wu K., 2000, Space Sci. Rev., 93, 611
Wu K., Chanmugam G., Shaviv G., 1994, ApJ, 426, 664 Wu K., Cropper M., Ramsay G., 2001, MNRAS, 327, 208 Wu K., Cropper M., Ramsay G., Saxton C., Bridge C., 2003, ChJAA, 3 Suppl., 235

Wynn G. A., King A. R., 1995, MNRAS , 275, 9

\section{APPENDIX A: COORDINATE SYSTEM}

Following Canalle et al. (2005), we adopt a curvilinear coordinate system $(u, w, \varphi)$ in which $\varphi$ is an azimuthal angle about the polar axis, $w$ is a path-length along a magnetic field line, and $u$ measures along an equipotential transverse to the field lines, effectively choosing the latitude of the fieldline's footprint on the stellar surface. The dipolar coordinates correspond to conventional polar coordinates according to

$u=\frac{\sin ^{2} \theta}{r}$ and

$w=\frac{\cos \theta}{r^{2}}$,

implying that

$w^{2} r^{4}+u r-1=0$. 

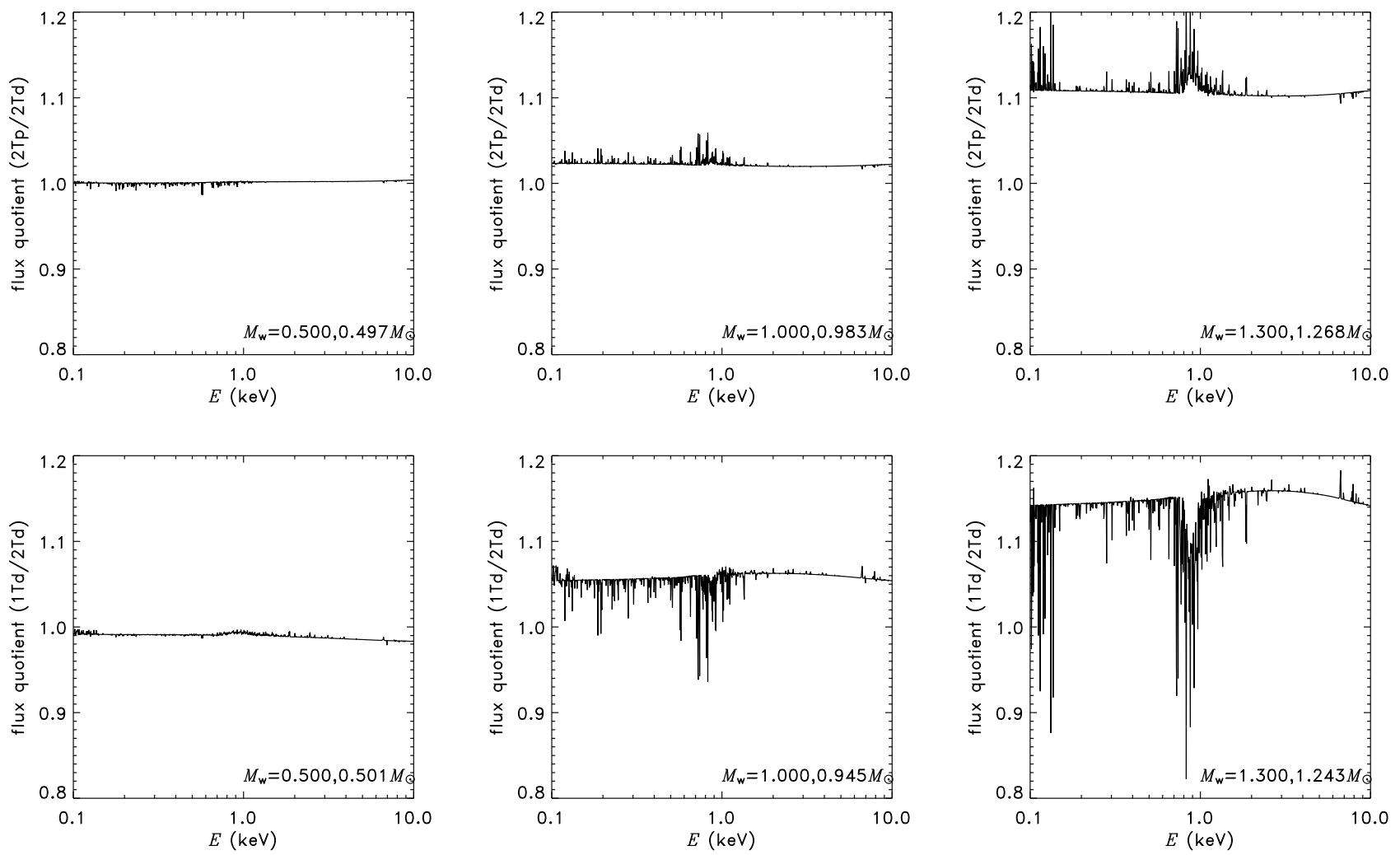

Figure 9. Matching the X-ray continua of two different accretion models requires different $M_{\mathrm{w}}$ values. The top row compares $2 \mathrm{~T}$ planar and dipolar models. The bottom row compares $1 \mathrm{~T}$ dipolar and $2 \mathrm{~T}$ dipolar models. Masses were adjusted to achieve approximately proportional continua (flat quotient profile). We have fixed $B_{*}=50 \mathrm{MG}, \dot{m}=2 \mathrm{~g} \mathrm{~cm}^{-2} \mathrm{~s}^{-1}$ and $\theta_{*}=0^{\circ}$. For low $M_{\mathrm{w}}$ (left panels) the inferred masses are similar; but for more massive cases the model masses differ appreciably.
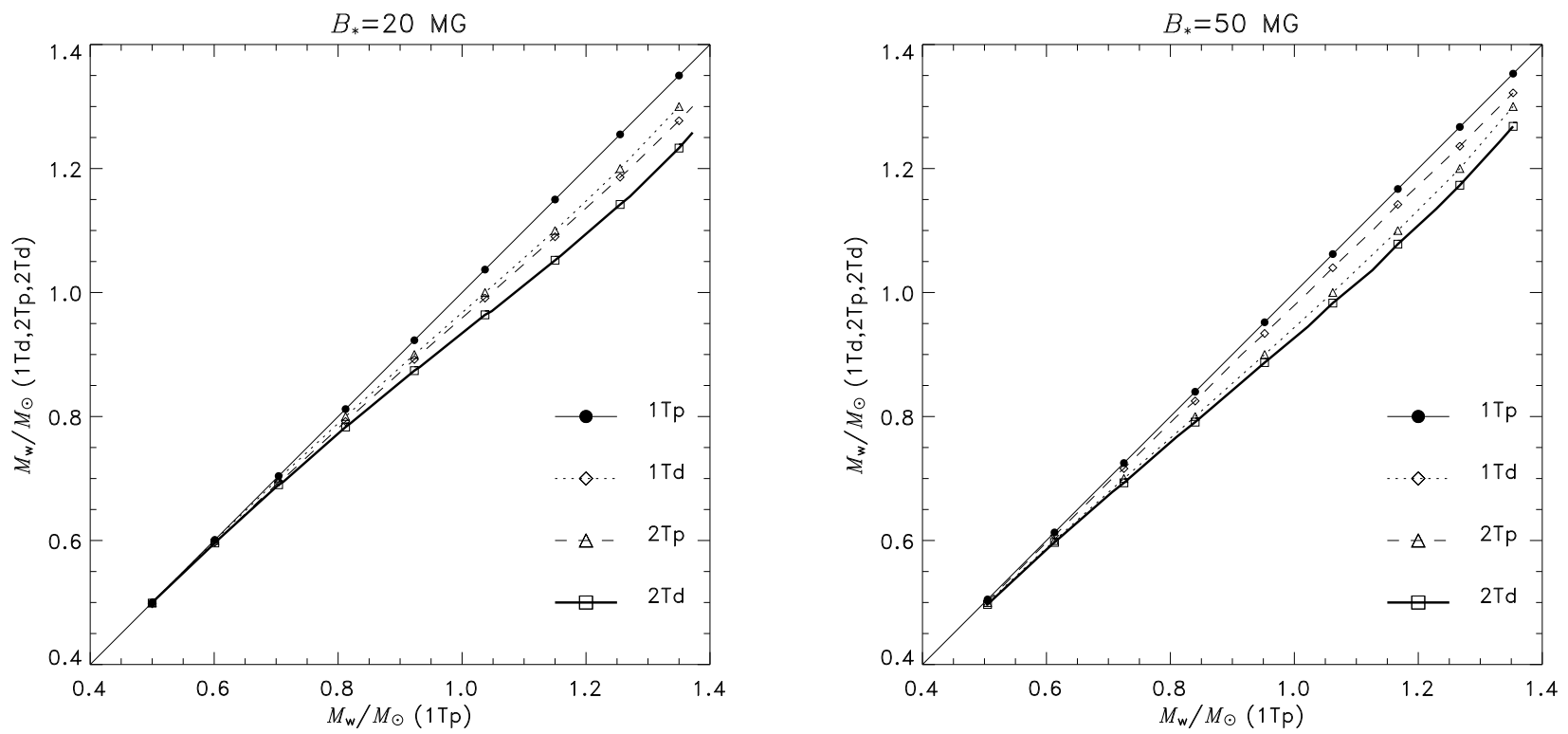

Figure 10. Masses inferred by fitting continua of $1 \mathrm{Tp}, 1 \mathrm{Td}, 2 \mathrm{Tp}$ and $2 \mathrm{Td}$ models to each other. Radially variable gravity, a dipolar accretion funnel and two-temperature effects yield harder spectra for lower $M_{\mathrm{w}}$ values. Here we have fixed the hot-spot size and $\dot{m}=$ $2 \mathrm{~g} \mathrm{~cm}^{-2} \mathrm{~s}^{-1}$. Symbols indictate $0.1 M_{\odot}$ steps in the $2 \mathrm{Tp}$ model; the curves are thin $/ \bullet(1 \mathrm{Tp})$, dotted $/ \triangle(2 \mathrm{Tp})$, dashed $/ \diamond(1 \mathrm{Td})$ and thick/ $\square(2 \mathrm{Td})$. 
Table 2. Sets of $M_{\mathrm{w}}$ values that give comparable spectral continua, calculated for the $1 \mathrm{Tp}, 1 \mathrm{Td}, 2 \mathrm{Tp}$ and $2 \mathrm{Td}$ models. The latter imply similar or lower masses. For these mass fits, we fix $\dot{m}=2 \mathrm{~g} \mathrm{~cm}^{-2} \mathrm{~s}^{-1}$ and $\theta_{*}=0^{\circ}$.

\begin{tabular}{cccc}
\multicolumn{4}{c}{$B_{*}=20 \mathrm{MG}$} \\
$1 \mathrm{Tp}$ & $1 \mathrm{Td}$ & $2 \mathrm{Tp}$ & $2 \mathrm{Td}$ \\
\hline 0.500 & 0.498 & 0.500 & 0.499 \\
0.601 & 0.597 & 0.600 & 0.596 \\
0.704 & 0.694 & 0.700 & 0.690 \\
0.812 & 0.792 & 0.800 & 0.783 \\
0.923 & 0.892 & 0.900 & 0.874 \\
1.037 & 0.991 & 1.000 & 0.964 \\
1.150 & 1.090 & 1.100 & 1.052 \\
1.255 & 1.186 & 1.200 & 1.142 \\
1.350 & 1.277 & 1.300 & 1.233 \\
& & & \\
\hline
\end{tabular}

\begin{tabular}{cccc}
\multicolumn{4}{c}{$B_{*}=50 \mathrm{MG}$} \\
$1 \mathrm{Tp}$ & $1 \mathrm{Td}$ & $2 \mathrm{Tp}$ & $2 \mathrm{Td}$ \\
\hline 0.505 & 0.502 & 0.500 & 0.497 \\
0.613 & 0.608 & 0.600 & 0.597 \\
0.725 & 0.716 & 0.700 & 0.693 \\
0.840 & 0.825 & 0.800 & 0.791 \\
0.952 & 0.934 & 0.900 & 0.887 \\
1.062 & 1.040 & 1.000 & 0.983 \\
1.167 & 1.142 & 1.100 & 1.078 \\
1.267 & 1.236 & 1.200 & 1.173 \\
1.353 & 1.322 & 1.300 & 1.268 \\
& & & \\
\hline
\end{tabular}

We scale the coordinate axes so that the stellar surface occurs at $r=1$. The transformation to Cartesian coordinates obeys

$$
\left[\begin{array}{l}
x \\
y \\
z
\end{array}\right]=\left[\begin{array}{c}
\sqrt{u r^{3}} \cos \varphi \\
\sqrt{u r^{3}} \sin \varphi \\
w r^{3}
\end{array}\right]
$$

The orthogonal unit vectors, $(\hat{\boldsymbol{u}}, \hat{\boldsymbol{w}}, \hat{\boldsymbol{\varphi}})$, vary spatially. Locally they transform into their Cartesian counterparts $(\hat{\boldsymbol{\imath}}, \hat{\boldsymbol{j}}$, $\hat{\boldsymbol{k}})$ according to

$$
\left[\begin{array}{c}
\hat{\mathbf{u}} \\
\hat{\mathrm{w}} \\
\hat{\varphi}
\end{array}\right]=\mathrm{U}\left[\begin{array}{c}
\hat{\imath} \\
\hat{\jmath} \\
\hat{k}
\end{array}\right] \text {. }
$$

where (see e.g. Arfken \& Weger 2001) the transformation matrix is

$\mathrm{U} \equiv\left[\begin{array}{ccc}\frac{1}{h_{1}} \frac{\partial x}{\partial u} & \frac{1}{h_{1}} \frac{\partial y}{\partial u} & \frac{1}{h_{1}} \frac{\partial z}{\partial u} \\ \frac{1}{h_{2}} \frac{\partial x}{\partial w} & \frac{1}{h_{2}} \frac{\partial y}{\partial w} & \frac{1}{h_{2}} \frac{\partial z}{\partial w} \\ \frac{1}{h_{3}} \frac{\partial x}{\partial \varphi} & \frac{1}{h_{3}} \frac{\partial y}{\partial \varphi} & \frac{1}{h_{3}} \frac{\partial z}{\partial \varphi}\end{array}\right]$

The coefficients $h_{1}, h_{2}$ and $h_{3}$ comprising the metric of the curvilinear coordinates system, are defined by

$h_{[1,2,3]}^{2} \equiv\left(\frac{\partial x}{\partial[u, w, \varphi]}\right)^{2}+\left(\frac{\partial y}{\partial[u, w, \varphi]}\right)^{2}+\left(\frac{\partial z}{\partial[u, w, \varphi]}\right)^{2}$,

with explicit functional forms given in Canalle et al. (2005). For a small fluid element following a magnetic field line, its transverse area varies as $h_{1} h_{3}$ in response to the converge of the field. The rate of convergence of the field lines, and thus the lateral compression of field-channelled inflow, can be expressed in terms of a function

$$
\mathcal{H} \equiv \frac{\partial}{\partial w} \ln \left(h_{1} h_{3}\right) \text {. }
$$

\section{APPENDIX B: NUMERICAL SOLUTION}

We find that it is convenient to express the post-shock structure in terms of a specific entropy variable,

$s \equiv P \rho^{-\gamma}=\left(\frac{h_{1} h_{3}}{C}\right)^{\gamma-1}(\xi-v) v^{\gamma}$,

which has a gradient equation,

$\frac{d s}{d w}=-\frac{(\gamma-1) \tilde{\Lambda} s}{(\xi-v) v^{5 / 2}}=-(\gamma-1)\left(\frac{h_{1} h_{3}}{C}\right)^{\gamma-1} \tilde{\Lambda} v^{\gamma-5 / 2}$.

Since the expression (B2) never changes sign, the spatial variation of $s$ is guaranteed to be monotonic throughout the post-shock flow. The maximum value of $s$ occurs at the shock; the specific entropy falls to zero at the stellar surface. Thus $s$ is a convenient replacement for $w$ in the role of independent, integration variable. Differential equations in terms of $d s$ are obtained by multiplying $d w / d s$ into (18), (19), (20) and (21) and then simplifying algebraic terms to reduce numerical round-off errors.

The strong shock boundary conditions imply that

$v_{\mathrm{s}}=\frac{1}{4 \sqrt{R_{\mathrm{s}}}}$

and

$\xi_{\mathrm{s}}=\frac{1}{\sqrt{R_{\mathrm{s}}}}$,

(Canalle et al. 2005), where we use a unit convention based on $C=1$, the stellar radius $R_{\mathrm{w}}=1$ and superficial escape velocity $V_{\mathrm{w}} \equiv \sqrt{2 G M_{\mathrm{w}} / R_{\mathrm{w}}}=1$. The shock values of $s$ and $P_{\mathrm{e}}$ follow from (B1) and (14), with $\sigma_{\mathrm{s}}$ treated as a free parameter. Assuming trial estimates of the shock location, we integrate inwards to $s \rightarrow 0$ and check for consistency between this inner radius and the stellar surface, $r=1$. A numerical root-finder iterates to a value of $R_{\mathrm{s}}$ that matches both boundary conditions.

This paper has been typeset from a $\mathrm{T}_{\mathrm{EX}}$ / $\mathrm{LT}_{\mathrm{E}} \mathrm{X}$ file prepared by the author. 